\title{
Dinámicas de integración educativa tras la COVID 19
}

Dynamics of educational integration after COVID.

\section{Magally Flores-Quispe}

Licenciada y Bachiller en Educación Secundaria, especialidad de Historia y geografía, Universidad Femenina del Sagrado Corazón, Maestra en Administración en la Educación, Universidad Cesar Vallejo, Coordinadora del área de Ciencias Sociales y docente de la Institución Educativa Estatal José María Arguedas, mfloresq17 @ucvvirtual.edu.pe, ORCID: 0000-0002-8571-455X, https://scholar.google.es/citations?hl=es\&user=E7HeBkYAAAAJ

\section{Revista Iberoamericana de la Educación Vol - Especial 12021 \\ e-ISSN: 2737-632x}

Resumen: Debido al COVID 19, la forma de trabajo de la escuela cambió radicalmente a nivel mundial y se trasladó al hogar junto con todos sus problemas de enseñanza y aprendizaje que debieron ser afrontados por las familias. El objetivo es analizar los diversos factores que permiten una adecuada integración entre la escuela y la familia para el beneficio del estudiante. La investigación se basa en una revisión sistémica teórica de la literatura correspondiente de diversos artículos científicos en materias de educación publicados en revistas indexadas durante los últimos seis años; abordando la problemática desde tres perspectivas: el involucramiento de los padres en el aprendizaje de sus hijos; los canales de comunicación entre la escuela y la familia y la participación de la familia en la gestión y actividades de la escuela. Llegando a la conclusión que la familia cumple un papel fundamental, pero no está preparada para responder a los nuevos retos que surgió por la pandemia, marcando una gran brecha socio-económica, la cual influyó en la comunicación entre estudiantes, docentes y padres de familia, siendo la escuela pública las más perjudicada.

Palabras clave: Familia, educación, escuela, comunicación, pandemia.

\section{Abstract}


Due to COVID 19, the school's way of working changed radically worldwide and moved to the home along with all its teaching and learning problems that had to be faced by families. The objective is to analyze the various factors that allow an adequate integration between school and family for the benefit of the student. The research is based on a theoretical systemic review of the corresponding literature of several scientific articles on education published in indexed journals during the last six years; approaching the problem from three perspectives: the involvement of parents in their children's learning; the channels of communication between the school and the family and the participation of the family in the management and activities of the school. The conclusion was that the family plays a fundamental role, but is not prepared to respond to the new challenges that arose due to the pandemic, marking a great socio-economic gap, which influenced the communication between students, teachers and parents, with the public school being the most affected.

Key words: Family, education, school, communication, pandemic.

\section{Introducción}

Uno de los grandes problemas que ha tenido la escuela es lograr una mayor participación de los padres de familia en la educación de sus hijos, ya que los consideran un aliado muy importante desde la educación temprana, porque influyen en su salud física y mental, las relaciones interpersonales e incluso su futuro éxito profesional (Park, 2004). El acompañamiento familiar es la base fundamental en el desarrollo de los procesos de socialización que invitan al encuentro de experiencias entre padres, madres e hijos en una zona de desarrollo próximo generando nuevos aprendizajes (Vygotsky, 1995). Permitiendo construir relaciones recíprocas de interacción, comunicación, conocimiento y comprensión para lograr un trabajo conjunto y coordinado 
entre la familia y la escuela para el bienestar del estudiante (Bronfenbrenner, 1987). En el presente siglo la escuela había dado un nuevo significado a su papel formativo estableciendo un contacto más cercano con las familias (Bolívar, 2006), coordinando diversas actividades entre padres de familia, docentes y dirección para el logro de aprendizajes; pero, la pandemia del COVID 19 cambió radicalmente la forma de trabajo de la escuela a nivel mundial con aulas vacías y aprendiendo en el camino las bondades de la educación a distancia en forma virtual, situación que sorprendió a los padres de familia, quienes al ver el cierre de los colegios se llenaron de dudas y temores al tener a sus hijos en casa y no saber cómo guiarlos en sus actividades escolares.

Por décadas la familia ha sido considerada como la unidad básica de la sociedad, en donde un conjunto de personas unidas por lazos de consanguinidad, afectivos o adopción, conviven en un mismo hogar y los adultos asumen la responsabilidad del cuidado respectivo de los hijos (Armas y Díaz, 2014 citado en Naranjo et al., 2020, p. 118). Concepto el cual está inmerso en importantes documentos como la carta magna de diversos países, que considera a la familia como una pieza fundamental para alcanzar el bienestar general, porque en ella recae la formación moral de los futuros ciudadanos. Según Dilleggi et al., (2019) las familias junto con la sociedad constituyen un todo, una organización con elementos vinculados que no pueden separarse y juntos con la escuela influyen en la formación psíquica del individuo. Porque ambos "son expertos y modelos educativos fundamentales de quienes quieren aprender" (Crisol y Romero, 2020, p. 47), siendo la educación un derecho humano y social que todo individuo debe disfrutar en igualdad de condiciones, la cual debe ser integral y de calidad, ya que posibilita el disfrute de los otros derechos esenciales (Pérez, 2014, citado en Tarazona et al., 2016, p. 176). 
Además, es importante resaltar que "la participación de los padres en la educación está asociada con una mejor asistencia a la escuela, puntajes más altos en matemáticas y lectura, más altas tasas de graduación en educación secundaria y menor grado de repitencia" (Larocque et al., 2011). Pero más allá de lo académico, las familias asumen un papel significativo en el proceso de aprendizaje y socialización de los niños y adolescentes y como su primera escuela es la principal transmisora de conocimientos producto de una serie de tradiciones, que van a influir en la toma de conciencia de su identidad personal, familiar y social (Aguilar, 2002). No hay duda que la familia tiene la gran "responsabilidad de favorecer el desarrollo humano imprimiendo desde los primeros años de vida la adopción de conductas que no representen riesgos psicosociales para sus miembros" (Paternina y Pereira, 2017, p. 435) siendo necesario una oportuna alianza entre la familia y la escuela porque ambas persiguen un mismo objetivo: educar de manera integral a los estudiantes (Romagnoli y Gallargo, 2018).

Esta alianza familia-escuela trae consigo tres importantes ventajas: Primero, el aumento de la motivación de los estudiantes, la cual es necesaria para un mejor aprovechamiento escolar, además contribuir a la adaptabilidad social y conducta en el aula; segundo, el incremento de habilidades parentales, referido a la interacción constante de los padres en el proceso educativo; tercero, la enseñanza más efectiva, la cual se cristaliza con la participación de los padres, los directivos de la escuela y profesores en las diversas actividades que conduzcan a una mejor calidad educativa (Epstein, 1992). Todas ellas necesarias porque no sólo abarca el campo académico sino también el social y tenemos que comprender que la escuela tiene por deber la socialización del niño, pero a veces las familias entran en guerra con la escuela cuando esta inculca normas, reglamentos y valores para sentar las bases de la vida social de los nuevos ciudadanos (Donatelli, 2004 citado en Pereira y Rivas, 2019) 
para lo cual es necesario utilizar diversos canales de comunicación para romper esas diferencias y fortalecer la confianza y el respeto mutuo.

Pero esta paralización generada por el COVID 19 ha implantado en las instituciones educativas una nueva forma de trabajo llamada zoomismo la cual se describe como un modo de producción a través de una clara inmovilidad en donde los gastos de electricidad, computadoras, internet, agua, incluso café son asumidos por los empleados desde su hogar (Estévez, 2020 citado en Morgado et al., 2020, p. 5), lo cual ha puesto en evidencia las grandes brechas que existen entre familias y escuelas para desarrollar su función educativa y ha evidenciado múltiples formas de exclusión (Tarabini, 2020, p.152), razón por la cual se reportan casos de deserción escolar, los cuales han aumentado debido a las carencias emocionales y materiales que atraviesan los múltiples hogares. Por esta razón el propósito de este artículo de revisión es brindar información con una visión amplia y definida sobre los diversos problemas existen y continúan enfrentando las familias y la escuela para trabajar en forma conjunta e incluso en esta nueva modalidad de educación a distancia debido a la pandemia.

\section{Materiales y métodos}

El presente trabajo de investigación es un artículo de revisión, el cual es "un estudio pormenorizado, selectivo y crítico que integra la información esencial en una perspectiva unitaria y de conjunto" (Vera, 2009, p. 1), elaborado siguiendo la metodología establecida por Guirao-Goris et al., (2008) para definir los objetivos de la revisión, realizar la búsqueda bibliográfica, organizar la información y redactar artículo. Tiene por objetivo de analizar los diversos diversos factores que han permitido una adecuada integración entre la escuela y la familia antes y durante la pandemia. 
Entre los criterios de inclusión, se valoró artículos que estén publicados en revistas científicas indexadas; que su investigación se haya realizado durante los años de 2014 al 2020 y que su escenario de estudio y población: estudiantes y familias se ubique en instituciones educativas básicas regulares; asimismo, se excluyeron de la búsqueda investigaciones con una antigüedad mayor a los siete años; publicadas en otros medios, como libros, manuales, blog; y estudios realizados en población universitaria, técnica y educación no formal.

El trabajo de investigación presentó momentos claves para la selección de artículos, se inicia a partir del mes de noviembre del 2020 con la búsqueda de palabras claves en el Tesauro de la Unesco: familia, educación, escuela y pandemia, lo que permitió una exploración; aproximadamente cerca de 100 artículos, en reconocidos portales de difusión de la producción científica como Scopus, ProQuest, Scielo, Dialnet, entre otros, así como buscadores electrónicos Google académico y Microsoft académico.

Tomando en cuenta los criterios de inclusión y exclusión se revisó 21 publicaciones comprendidas entre los años 2014 y 2019 para comprender la problemática de relación familia y escuela antes de la pandemia y luego 19 publicaciones del 2020 para recabar datos dentro del contexto educativo durante la emergencia sanitaria del COVID 19. De ellas se pudo llegar a diversas fuentes primarias y secundarias que han investigado sobre la relación: escuela y familia logrando tener un marco referencial de cerca de 51 fuentes bibliográficas provenientes de revistas indexadas, libros y otros; abordando la problemática desde tres perspectivas: el involucramiento de los padres en el aprendizaje de sus hijos; los canales de comunicación entre la escuela y la familia y la participación de la familia en la gestión y actividades de la escuela. 


\section{Resultados}

Las investigaciones revisadas consideran a la escuela, como la institución especializada en transmitir conocimientos, habilidades y destrezas, así como formar actitudes, disposiciones y caracteres; que junto con otras instituciones sociales como la familia contribuyen al proceso de socialización de los niños, niñas y jóvenes" (Tarabini, 2020, p. 146). Dado que el hogar contribuye en la formación del niño y el adolescente, Martiniello (1999) estableció en una taxonomía en cuatro categorías la forma de cómo pueden las familias participar en la escuela: Primero como responsables de la crianza, los padres velan por el cuidado y protección de los niños, promoviendo su asistencia a la escuela con las condiciones necesarias; segundo, como maestros, supervisando, facilitando y reforzando el desarrollo de las tareas escolares; tercero, como agentes de apoyo a la escuela, contribuyendo en la mejora de las condiciones del proceso de aprendizaje a nivel económico, de tiempo, aportando ideas y materiales; y cuarto, como agentes con poder de decisión, participando en las asociaciones de padres de familia, conociendo cómo opera la escuela y las políticas que la gobiernan. (Aguirre et al., 2019)

Esta propuesta fue tomada en cuenta por diversos autores, destacando Sarmiento y Zapata (2014) en su marco conceptual presentando cuatro dimensiones sobre la participación de la familia en la escuela: el involucramiento de los padres en el aprendizaje de sus hijos; los canales de comunicación entre la escuela y la familia; el involucramiento de la familia en la gestión y el desarrollo de actividades de la escuela; y finalmente la alianza entre las familias, la escuela y la comunidad. Además nos permite tener un panorama más amplio sobre los factores que influyen en la integración escuela y familia porque parte del hogar y termina con la sociedad, además involucra a todos los actores de la comunidad educativa tomando en cuenta diversas características sociodemográficas: el nivel 
educativo y la lengua materna de la madre; quien mayormente se encarga de la educación de los hijos y las que acuden al colegio ante cualquier problemática o llamado de la escuela, así como el nivel socioeconómico de la familia y la zona donde se ubica el hogar; los cuales son necesarios para cubrir las necesidades básicas para el desarrollo del estudiante. (Barros Bastidas \& Turpo Gebera, 2018)

\section{Primera dimensión: Los padres de familia y el aprendizaje de sus hijos}

En la primera dimensión: Los padres de familia y el aprendizaje de sus hijos, se encontró trabajos provenientes de países sudamericanos y de España, de los cuales cuatro corresponden al año 2020 y los demás entre los años 2019 y 2016. Los estudios tuvieron como objetivo analizar la forma cómo la pandemia afectó a las familias, teniendo que asumir el rol de educadores desde el hogar para el cual no estaban preparados; entre ellos Vázquez et al., (2020) quien realizó un estudio cualitativo entrevistando a más de 20 estudiantes y padres de familia para recoger sus primeras vivencias educativas fuera de la escuela durante el confinamiento y como parte de un estudio macro sobre la coyuntura actual.

Mientras que Cabrera (2020) presentó un análisis exhaustivo en España con las cifras de los estudiantes directamente afectados por el cierre escolar, describiendo la tipología de los hogares por los dispositivos electrónicos y a la conexión a internet que poseen, resaltando el impacto negativo en las familias menos favorecidas y el aumento de la desigualdad de oportunidades educativas; por su parte Kohan (2020) describió la forma como el gobierno de Brasil afrontó la pandemia del COVID-19 y cómo esta política educativa afectó a las familias y el aprendizaje de los estudiantes, finalmente ValeroCedeño et al. (2020) analizó los retos que la educación virtual presentó para los docentes y estudiantes de cualquier nivel educativo por la pandemia 
debido a la poca familiaridad con los sistemas electrónicos, dando por descubierto las grandes brechas digitales entre la educación pública y privada. Sobre el comportamiento de la escuela antes de la pandemia, Mayorquín y Zaldívar (2019) analizaron la relación de la participación de los padres de familia con el aprovechamiento escolar de los estudiantes; mientras que Renta et al., (2019) presentaron evidencias científicas sobre el aumento de la motivación de los estudiantes en riesgo de exclusión mediante la participación de la familia, considerándola como un principal agente motivador para que el niño o adolescente no abandone su preparación académica; por su parte Villalobos et al., (2017) por medio de entrevistas investigó la influencia de la escuela y la familia en relación con el alcance del logro académico, resaltó la necesidad de que la escuela propicie acciones que convierta a padres, madres y acudientes en agentes activos del proceso de formación; finalmente Tarazona et al., (2016) analizaron la interrelación familia - escuela en el Ecuador mediante un estudio comparativo entre los marcos de desarrollo en dicha área con los planteados por la Organización de las Naciones Unidas para la Educación, la Ciencia y la Cultura (UNESCO), destacaron la importancia del binomio escuela y familia en el proceso de enseñanza, así como su influencia positiva en el aprendizaje de los estudiantes. (p. e.: tabla 1)

\section{Segunda dimensión: La comunicación entre la escuela y la familia}

En la segunda dimensión: La comunicación entre la escuela y la familia, se revisó 13 artículos de los cuales diez corresponden al año 2020 y tres a los años entre los años 2018 y 2016. Las investigaciones de diversas partes del mundo, como Garduño (2020) y Scalabrin y Côco (2020) se preocuparon por analizar las políticas educativas de gobierno que se utilizaron para afrontar la la pandemia, siendo considerado por Morgado et al. (2020) como un cambio 
de paradigma en la enseñanza a distancia; generando grandes desafíos para la escuela por el uso de diversas tecnologías de la información y comunicación (Hurtado, 2020); dado a que no se consideró el impacto económico con el cierre de varios negocios y pérdida de empleos, repercutiendo en el sistema educativo y agudizando los problemas de comunicación que ya existía entre la escuela y los padres de familia (Jarquín, 2020).

Por su parte Naranjo et al., (2020) también aprovechan la coyuntura mundial y utilizando una metodología descriptiva, no experimental y retrospectiva durante el periodo febrero a junio analizando los efectos de la pandemia en la familia y en la sociedad ecuatoriana; mientras que Ruíz (2020) reflexionó sobre la relación entre la investigación educativa, la práctica profesional y la toma de decisiones durante la pandemia; ambos al igual que Russo et al. (2020) identificaron un reordenamiento en la educación escolar y como se ampliaron las desigualdades educativas; siendo investigado por Tarabini (2020) desde un enfoque de la justicia educativa y social; llegando a la misma conclusión con Velásquez (2020) que se amplió la brecha de exclusión social y digital en la escuela privada y pública, siendo la más afectado esta última. Antes de la pandemia, las investigaciones realizadas coinciden en la necesidad de optimizar la comunicación con los padres de familia; con un estudio de enfoque cuantitativo Arnaiz y Chamseddine, (2020) analizó el conocimiento que tienen los docentes sobre la vida del alumno y el tipo de relaciones que mantienen ambas partes, descubriendo grandes problemas en la comunicación; mientras que Lupion (2018) dio a conocer las diferentes estrategias que la escuela puede utilizar para trabajar con las familias y reforzar la necesidad de mantener una comunicación con las familias de los estudiantes; por su parte Macia (2018) presentó un plan estratégico de mejora en donde consideró las características y necesidades de las familias siguiendo la estructura de las 6W del periodismo: qué, quién, dónde, cómo, cuándo y 
por qué, para optimizar la comunicación entre los padres y la escuela. Finalmente, Lv, et al. (2016) exploró la relación entre el rendimiento académico y el bienestar emocional (medido como afecto positivo y negativo) de los niños de la escuela primaria y el efecto moderador de la comunicación. (p. e.: tabla 2)

\section{Tercera dimensión: Participación de la familia en la gestión}

En la tercera dimensión: Participación de la familia en la gestión y actividades de la escuela, se revisó diez artículos de los cuales cuatro corresponde al año 2020 y los otros seis se ubican entre los años 2018 y 2015; también se encontró un artículo de Domingo et al. (2010) el cual es mencionado por investigadores y organismos gubernamentales en educación de los países latinoamericano por proponer líneas de trabajo para mejorar la relación escuela - familia.

Estos trabajos tuvieron como objetivo analizar la forma de trabajo de la escuela con las familias durante la pandemia, Como Belmonte y BernárdezGómez (2020) quienes recopilaron y contrastaron las percepciones sobre la educación, formal o no formal, llevada a cabo en los hogares, a raíz del confinamiento. Mientras que Hurtado (2020) se propuso identificar los nuevos desafíos que tuvo que asumir la escuela en este nuevo escenario, entre ellos su distanciamiento con las familias. Es por ello que Arnaiz y Chamseddine (2020) describió el conocimiento del centro que poseen los progenitores y el tipo de relaciones personales que mantienen ambas partes; mientras que Romero (2020) contextualizó las vivencias actuales que permean y constituyen las experiencias formativas de familias y estudiantes; para reconocer los problemas y retos por vencer para lograr un trabajo coordinado en favor de los estudiantes. 
Pero antes de la pandemia Rodríguez y Vasconcelos, (2019), evaluaron el impacto de las prácticas de integración familia-escuela como: tarea, reunión de padres, expectativas de la escuela con respecto a los padres o tutores y estrategias de comunicación de la escuela con la familia en el aprendizaje de los estudiantes; mientras que, con un cuestionario sencillo y casi lúdico Pereira y Rivas (2019) determinaron cómo los padres y madres son actores indispensables en el éxito académico de los niños de un centro de enseñanza pública de primaria. Por su parte, Razeto (2018) analizó las prácticas utilizadas por las escuelas para incrementar la participación de los padres en la educación, debido a su impacto positivo en el estudiante; por esta razón Ruvalcaba-Romero, et al., (2018) realizó pruebas de correlación para identificar el nivel predictivo de las relaciones, la comunicación afectiva con los padres y la generación de conductas pro sociales sobre la experimentación de emociones positivas; Mo y Singh (2015) examinaron el efecto de las relaciones y la participación de los padres en el compromiso cognitivo, emocional y conductual de los estudiantes en la escuela; finalmente, León y Fernández (2017) presentaron un estudio para diseñar y validar un instrumento con cuatro dimensiones: relación, apoyo pedagógico, participación. (p. e.: tabla 3)

\section{Discusión}

\section{Los padres de familia y el aprendizaje de sus hijos}

La pandemia ha golpeado a millones de familias que no sólo enfrentan la emergencia sanitaria con el confinamiento en los hogares sino también el cierre de las escuelas, las cuales para poder atender a los estudiantes plantea nuevas exigencias educativas marcando grandes desigualdades sociales por su falta de disposición al internet o no poseer algún tipo de ordenador para poder recibir sus clases virtuales, brecha que se acrecienta si comparamos el 
tipo de institución pública y privada demostrando esta última estar mejor preparada en docentes y hogares para conectarse a la nueva modalidad virtual (Cabrera, 2020; Kohan, 2020). Incluso el Instituto Apoyo Perú, señaló “que ante las limitaciones que tienen los hogares producto de las repercusiones económicas de la pandemia se deben ofrecer alternativas acordes con esta realidad" (Morales, 2020 citado por Valero-Cedeño et al., 2020, p.1207), no hay duda que la escuela se trasladó al hogar, junto con sus problemas de enseñanza y aprendizaje los cuales deben ser afrontados por las familias las cuales no están preparadas para responder y mucho menos para entender la forma como debe orientar académicamente a sus hijos.

Una de las principales problemáticas que han tenido que enfrentar los hogares es la poca capacidad por parte del padre de familia para apoyar en las cuestiones académicas de sus hijos y la falta de habilidades de autoestudio en los estudiantes (Vázquez et al., 2020, p. 21), bajo el argumento del exceso de trabajo, la gran mayoría de los padres no se ha involucrado en las labores escolares y tampoco se ha fomentado desde el hogar hábitos de estudio que les permita a los niños y jóvenes a trabajar solos sin la supervisión de un adulto. La única forma que la educación virtual tenga éxito durante el período de la pandemia, es con la colaboración de las familias la cuales deben monitorear el desempeño y el comportamiento de sus hijos, respetando su sentido de identidad y las necesidades acordes a su edad, pero en especial ayudarlos a mejorar sus capacidades de autogestión y autoaprendizaje (Valero-Cedeño et al., 2020).

Está comprobado que la familia es el principal soporte en la educación y con ella se logra los mejores resultados académicos cuando apoya, estimula y motiva constantemente a sus hijos, (Renta et al., 2019; Mayorquín y Zaldívar, 2019). Además, al establecer metas realistas dentro de sus capacidades y posibilidades les permite llegar al final de una etapa porque se tiene la firme 
decisión de hacer algo (Villalobos et al., 2017) transmitiendo valores como: optimismo, responsabilidad y perseverancia para alcanzar objetivos y labrarse un futuro con un proyecto de vida claro, objetivo y viable. Incluso Tarazona, et al., (2016) señaló que: "la implicación de la familia en el proceso educativo es crucial si se desea disminuir el indicador de abandono de los estudios" (p.178) un punto más a favor de la familia, si deseamos disminuir la deserción escolar.

(p. e.: figura 1)

\section{La comunicación entre la escuela y la familia.}

Durante el tiempo de pandemia, ha sido necesario el uso de diversos medios tecnológicos para acceso a la información y establecer la comunicación necesaria con los estudiantes y padres de familias, así como también entre los profesionales de la educación (Naranjo et al., 2020, p. 133) pero el sector educativo es considerado como una empresa basada en las relaciones y el acto pedagógico es un momento de interacción y compartir el cual se ha visto en peligro al recurrir a la tecnología para mantener un frío acercamiento entre estudiantes y padres familia (Morgado, et al., 2020 p. 6), porque ha sido distante debido a los pobres recursos y condiciones de las familias que al no contar con los medios suficientes se limitó su comunicación a un simple envío de tareas y la recepción de documentos y trabajos realizados en forma solitaria por los estudiantes.

No hay duda que la pandemia derrumbó la tesis de los organismos de la globalización que señalaban que la tecnología y el internet lograrían llevar educación a diversos lugares lugares del mundo; porque lo que generó la enseñanza virtual es excluir a diversos sectores más vulnerables de la zona rural y urbana, ampliando la brecha de la desigualdad (Velásquez, 2020; Jarquín, 2020). Es por ello que en varios hogares; por la falta de una escuela virtual, los madres y padres se convirtieron en docentes y les tocó regular las 
tareas, supervisar si los hijos se conectan a los programas de televisión, trabajan los libros de texto o envían las evidencias en carpetas de experiencias, aumentando así la tensión familiar del confinamiento porque la casa no es la escuela; los padres de familia no son maestros y la comunicación por los medios electrónicos no equivale al ambiente del aula. (Garduño, 2020, p. 21-22) Además no hay olvidar que a pesar de sus grandes esfuerzos los padres de familia no cuentan con los recursos de formación, el tiempo adecuado, las condiciones materiales y la vocación necesaria para asumir este nuevo rol (Ruiz, 2020; Russo et al., 2020).

Es esencial una óptima comunicación para establecer una buena relación entre la familia y la escuela (Lupion, 2018) y lograr un óptimo rendimiento académico del estudiante, por ello se recomienda a los padres estar pendientes de las calificaciones, en el quehacer educativo y acudir a la escuela ante cualquier duda; asimismo el docente debe estar dispuesto a atenderlos para que juntos puedan encontrar soluciones ante cualquier problema que no permita un buen desenvolvimiento académico del niño o joven. Incluso en esta coyuntura actual de pandemia, uno de los retos de la escuela es establecer una comunicación efectiva no solo con los estudiantes, sino también con los padres, a los cuales se les debe brindar un acompañamiento desde el ámbito emocional, ya que para muchas familias la responsabilidad de la educación recaía completamente en la escuela. (Hurtado, 2020)

La comunicación entre los padres y la escuela modera la relación del afecto con el rendimiento académico, porque los progenitores al darle importancia en comprender la capacidad académica de sus hijos pueden modificar o ajustar su comportamiento de crianza (Lv, et al., 2016). Concuerdan con esta idea Scalabrin y Côco (2020) e indican que a pesar que es todo un desafío, es necesario intensificar la aproximación de la familia con la escuela desde la educación infantil. Además, Macia (2018) señala que, si los docentes 
aprovechan los encuentros con las familias, como sus canales de comunicación para transmitir aspectos positivos de los estudiantes y todos sus logros obtenidos, esto puede influir en la motivación para obtener mejores resultados y continuar con sus estudios. En sí, ambas partes deberían siempre fortalecer los logros del niño, adolescente o joven ello le ayudaría a tener confianza para afrontar nuevos retos, ser creativo y creer en sus ideas. Porque según Arnaiz y Chamseddine, (2020, p. 128) al establecer procesos de comunicación viables y alejados del modelo tradicional se favorece la confianza mutua, como motor esencial para una relación estable y de calidad. (p. e.: figura 2)

\section{Participación de la familia en la gestión y actividades de la escuela}

Es lamentable apreciar cómo va desvaneciendo la presencia del padre familia en la escuela, conforme su hijo crece y es promovido a los años superiores, "menos de la mitad de los padres de los estudiantes de secundaria participan activamente en programas y actividades escolares" además la mayoría se centran en los niveles académicos inferiores como educación temprana y nivel primaria (Johnston, 1998 citado en Mo y Singh, 2015, p. 2). Los directores valoran la participación parental, pero pierden su encanto cuando genera dificultades, además no se aprecia un programa de relación familiaescuela instalado, que promueva un proceso de planificación estratégica de las formas de colaboración proactiva y que fuesen adaptables al cambio (Egido, 2015, citado en Razeto, 2018), antes de la pandemia solo se ha trabajado con estrategias muy tradicionales y de poca innovación siendo las actividades festivas las únicas que convoquen a una mayor asistencia de las familias pero que no influye en el aprendizaje del niño. Según Hurtado (2020) la familia y la escuela tienden a observarse unidireccionalmente como si no compartiesen propósitos educativos comunes debido a que a principios del 
siglo XX empezaron a distanciarse, delegando los padres la educación de sus hijos exclusivamente a los docentes (p.182).

Aunque a veces no encontremos esa sintonía esperada por posturas diferentes que puedan generar conflictos la familia y escuela deben sumar esfuerzos en una misma dirección porque "la educación no es exclusiva de ninguno de ellos, ni se puede articular con coherencia sin una adecuada integración de esfuerzos y responsabilidades entre los mismos" (Domingo et al., 2010 p. 112). Se conoce que los padres y madres tienen una gran acumulación de responsabilidades por los quehaceres del hogar y sus largas horas de trabajo, lo que les impide ir a la escuela por carecer de tiempo. (Llevot y Bernard, 2015 citado en Arnaiz y Chamseddine 2020 p. 117) por este motivo, es necesario establecer canales de comunicación efectiva y afectivas, con sentimientos de aceptación y comprensión entre padres e hijos para promover la autonomía e individualidad (Cumsille et al., 2015; Meier y Oros, 2012 citados en Ruvalcaba-Romero et al., 2018, p. 185) para lograr conquistar el interés por las actividades escolares y su progresiva integración e interacción con la propia escuela.

Es necesario promover la participación formal e informal de los padres de familia; con invitaciones a diversos proyectos con actividades curriculares y extra-curriculares para proporcionarles información sobre el desarrollo social y emocional de sus hijos como además buscar su aportes y orientación en las decisiones educativas (Mo y Singh, 2015). Porque tal como Domingo et al. (2010) señalaron: "Debemos esforzarnos todos por transmitir la idea de la coparticipación, la necesidad de promover la colaboración entre la familia y la escuela, para afrontar juntos los retos educativos que nos plantea el futuro" (p.114) y se puede empezar modificando las formas de comunicación entre la escuela y la familia para que ambos compartan una misma visión de la escuela 
e incorporen acciones conjuntas para solucionar los problemas de aprendizaje del estudiante. (p. e.: figura 3)

Además, también la escuela debe reconocer que existe una diversidad de familias para no caer en la exclusión, porque si somos conscientes de la situación crítica que atraviesa el sector económico, las diferencias sociales se han incrementado y nuestros estudiantes de la escuela pública e incluso la privada, cada vez tienen poca disponibilidad de contar con los materiales de estudio que demanda la era digital. Y si nos referimos al área rural Romero, (2020) nos relata que este aislamiento social ha borrado los lazos colectivos y estrategias solidarias debido al temor por el contagio que nos obliga a la vida dentro del hogar y que pone en manifiesto las desigualdades ya existentes respecto de dificultades de acceso al trabajo, a la comida, al agua y señal de internet diaria porque se debe recorrer grandes distancias para conectarse además de desarrollar las tareas escolares en solitario. Por este motivo es importante que todas las piezas de la estructura comunitaria, continúen unidas, apoyándose en las iniciativas educativas de valor, con la finalidad de salvar un presente que se ha visto transformado dramáticamente por la pandemia, necesitamos una sociedad que pueda hacer frente a todo tipo de desavenencias actuales y futuras, procurando preservar el verdadero valor de la educación garantizando su función social de calidad, ante esta difícil y excepcional situación (Belmonte y Bernárdez-Gómez, 2020) que estamos afrontando y esperamos que termine muy pronto.

\section{Conclusiones}

La familia cumple un papel fundamental en el logro de los aprendizajes de los estudiantes porque no solo provee los recursos necesarios, sino que transmiten valores y aumenta la motivación que necesitan de los niños y jóvenes para un mejor aprovechamiento escolar, además favorecen a la 
adaptabilidad social y conducta en el aula. Por lo tanto, la escuela y la familia deben trabajar de manera conjunta en actitud de acción, corrección y mejora en las diversas dificultades que puedan generarse porque no todo se aprende en la escuela, el hogar y la comunidad contribuye en la formación del niño y el adolescente, lo que se debe evitar es un cruce de información desigual para no arar en desierto y lograr metas comunes en favor del estudiante para potenciar sus habilidades y competencias académicas que les permitan desenvolverse en el futuro.

Pero, se está apreciando muy poca participación de la familia en aprendizaje de sus hijos por diversos factores como: la disfuncionalidad en el hogar, el cual debe entenderse cuando los apoderados no cumplen con sus responsabilidades frente a sus hijos; los problemas económicos, que crea brechas sociales y mantienen a los padres lejos del hogar, llegando a casa sólo con deseos de descansar por el arduo trabajo; y el bajo grado académico de la madre y el padre que no les permite orientar adecuadamente a sus hijos en la tarea escolar, rol que se vio incrementado generando mayores problemas en el proceso de aprendizaje.

Con la pandemia los docentes han descubierto nuevos canales de comunicación mediante el uso de la tecnología como el WhatsApp, zoom, redes sociales u otros, como también una simple llamada telefónica que nos permitan acortar distancias entre maestros, estudiantes y padres; pero es necesario establecer nuevas formas de comunicación: las citaciones deben ser para comunicar logros y descubrir problemas que no permiten mejorar el aprendizaje de los estudiantes, como también brindar un soporte emocional y de estrategias de enseñanza - aprendizaje a los padres de familia; debido a que no estaban preparados para asumir el rol de educadores, como además para motivar su presencia en la escuela. Estos mensajes positivos permiten un 
trabajo conjunto, en donde todos asumen una responsabilidad de acuerdo a sus funciones como estudiante, padre o educador.

En los últimos años se ha generado una serie de políticas de reforma escolar que promueve la participación de los padres en la escuela, motivando una atención personalizada y en forma colectiva a los padres de familia por parte del tutor, sin olvidar las actividades de puertas abiertas que organiza la escuela para exponer los logros de los estudiantes y otras festividades de acuerdo a las características de la comunidad. Pero, así como hay que propiciar un acercamiento, se recomienda tener cuidado de ocasionar todo lo contrario, hay que evitar una serie de discriminaciones como elogiar a los padres que lograron encajar con un supuesto perfil esperado por la escuela; hay que dejar de ver a los padres como un proveedor financiero y una madre dedicada al cuidado del niño y las tareas del hogar, hay que adaptarnos a los nuevos tiempos con padres y madres alejados del hogar por tener la responsabilidad de mantener sus hogares.

Finalmente, a pesar de que la escuela reconoce la importancia de la participación de los padres de familia en la gestión y diversas actividades aún no se logra desarrollar estrategias que permitan incrementar el interés y conseguir una asistencia masiva a las reuniones durante el año. Se nota una mayor participación en los grados iniciales, pero este interés va decayendo en los grados superiores, en donde el estudiante adolescente pierde el acompañamiento del padre o la madre y si no ha aprendido a estudiar solo, es fácil que pierda interés por las tareas de la escuela. Sería recomendable realizar a futuro una serie de investigaciones cualitativas y cuantitativas sobre qué han aprendido los estudiantes en sus clases a a distancia por motivo de la pandemia; cuántos realmente han estado conectados en sus clases remotas; cuántos han abandona sus estudios por problemas económicos; así como la 
familia logró o no seguir apoyado a sus hijos adolescentes en sus estudios durante la pandemia.

\section{Referencias}

Aguilar, M. (2002). Familia y escuela ante un mundo en cambio. Revista contextos de educación, 5, 202-215.

Arnaiz, P., y Chamseddine, M. (2020). Relación entre familias migrantes y profesorado: un camino en construcción. Revista Científica: Revista Española De Orientación y Psicopedagogia, 31(2), 115-131. https://doi.org/10.5944/reop.vol.31.num.2.2020.27989

Aguirre Cardona, L. A., López C, J. E., \& Villamizar Gómez, D. F. (2019). Revisiones y reflexiones en la educación física: un camino de lo conceptual a lo investigativo en la escuela.

Barros Bastidas, C., \& Turpo Gebera, O. (2018). Factors influencing the scientific production of university professors: a systematic review. Factores Que Influyen En La Producción Científica de Los Docentes Universitarios: Una Revisión Sistemática., 11(22), 225-234. http://10.0.85.43/pensam.v11i21-

1.276\%0Ahttps://ezproxy.uniandes.edu.co:8443/login?url=http://search.ebsc ohost.com/login. asp $x$ direct=true $\& \mathrm{db}=$ fua $\& A N=141223533 \&$ lang=es $\&$ site $=$ ehost-live

Belmonte, M., y Bernárdez-Gómez, A. (2020). Respuesta social al estado del aislamiento por coronavirus, percepciones sobre la educación. Knowledge Online, 3, 30-49. https://doi.org/10.25112/rco.v3i0.2326 
Bolívar, A. (2006). Familia y escuela: dos mundos llamados a trabajar en común. Revista de Educación, 339, 119-146. https://tinyurl.com/y8xp2uor Bronfenbrenner, U. (1987). La ecología del desarrollo humano. Paidós.

Cabrera, L. (2020). Efectos del coronavirus en el sistema de enseñanza: aumenta la desigualdad de oportunidades educativas en España. Revista de Sociología de la Educación - RASE, 13(2), 114-139. http://dx.doi.org/10.7203/RASE.13.2.17125

Cando, F., Herrera, M., Drobchak, B., y Drobchak, V. (2017). Escribir y publicar artículos científicos en revistas indexadas: paso a paso. Universidad Técnica de Cotopaxi.

Contreras, C., Calvachi, L., Leon, A., \& Restrepo, G. (2017). Familia y escuela, juntos construyendo convivencia y paz. Evaluación de una experiencia de intervención. Revista Logos, Ciencia y Tecnología, 9(2), 5563. http://dx.doi.org/10.22335/rlct.v9i2.478

Crisol, E., y Romero, M. (2020). El liderazgo inclusivo como estrategia para evitar el abandono escolar: opinión de las familias. Educatio Siglo XXI, 38, 38(2), 45-66. https://doi.org/10.6018/educatio.414871

De León Sánchez, B. (2011). La relación familia-escuela y su repercusión en la autonomía y responsabilidad de los niños/as. XII Congreso Internacional de Teoría de la Educación. https://extension. uned. es/archivos_publicos/webex_actividades/5385/repercusiones8.pdf.

Dilleggi, E., Rosa, A., y Santos, P. (2019). Family functioning and environmental resources offered by families of children with mental $\begin{array}{llll}\text { disorders. } & \text { Salud } & \text { 235-242. }\end{array}$ 
http://www.scielo.org.mx/scielo.php?script=sci_arttext\&pid=S018533252019000500235

Domina, T. (2005). Leveling the home advantage: Assessing the effectiveness of parental involvement in elementary school. Sociology of education, 78(3), 233-249. https://doi.org/10.1177/003804070507800303

Domingo, J., Martos, M., y Domingo, L. (2010). Colaboración familia escuela en España: retos y realidades. Revista de Estudios y Experiencias en Educación, 9(18), 111-133. https://www.redalyc.org/pdf/2431/243119055008.pdf

Epstein, J. (1992). School and family partnerships. Report $N^{\circ} 6$. https://eric.ed.gov/?id=ED343715

Garduño, T. (2020). La pandemia y la utopía: hacia nuevos paradigmas

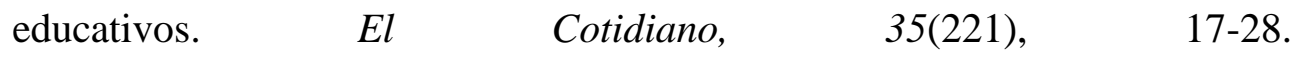
https://search.proquest.com/scholarly-journals/la-pandemia-y-utopía-hacianuevos-paradigmas/docview/2462681312/se-2 ?accountid=37408

Hurtado, F. (2020). La educación en tiempos de pandemia: los desafíos de la escuela del siglo XXI. CIEG, Revista arbitrada del centro de investigación y $\begin{array}{lll}\text { estudios } & \text { gerenciales, } & \text { 176-187. }\end{array}$ http://revista.grupocieg.org/publicacion/revista-cieg-n-44-julio-agosto-2020/ Jarquín, M. (2020). Educación en tiempos virales: SARS CoV-2, sistemas educativos y nueva escuela mexicana. El Cotidiano, 35(221), 39-52. https://search.proquest.com/scholarly-journals/educación-en-tiemposvirales-sars-cov-2-sistemas/docview/2462681297 
Kohan, W. (2020). Tempos da escola em tempo de pandemia e necropolítica.

$\begin{array}{llll}\text { Práxis } \quad \text { Educativa } & \text { (Brasil), } & 15, & 1-9 .\end{array}$ https://doi.org/10.5212/PraxEduc.v.15.16212.067

Larocque, M., Kleiman, I., y Darling, S. (2011). Parental involvement: the missing link in school achievement. Preventing School Failure: Alternative Education for Children and Youth, 55(3), 115-122., 55(3), 115-122. https://doi.org/10.1080/10459880903472876

León, V., y Fernández, M. (2017). Diseño y validación de un instrumento para evaluar la participación de las familias en los centros educativos. Revista Española De Orientación y Psicopedagogía, 28(3), 120-137. https://search.proquest.com/scholarly-journals/diseño-y-validación-de-uninstrumento-para/docview/2033735350/se-2?accountid=37408

Lupion, G. (2018). A relação escola-família e a organização do trabalho pedagógico na educação infantil. Revista Ibero-Americana de Estudos em Educação, $\quad$ Araraquara, 13(3), 1193-1209. https://doi.org/10.21723/riaee.v13.n3.2018.9778

Lv, B., Zhou, H., Guo, X., Liu, C., Liu, Z., y Luo, L. (2016). The relationship between academic achievement and the emotional well-being of elementary school children in China: The moderating role of parent-school communication. Frontiers in Psychology, 7(948). https://doi: 10.3389/fpsyg.2016.00948

Macia, M. (2018). Aproximación teórica a la comunicación familia-escuela: Estrategias de mejora. Ehquidad, 10, 89-112. http: //dx.doi.org/10.15257/ehquidad.2018.0010 
Martiniello, M. (1999). Participación de los padres en la educación: Hacia una taxonomía para América Latina. Harvard University.

Mayorquín, E., y Zaldívar, A. (2019). Participación de los padres en el rendimiento académico de alumnos de primaria. RIDE. Revista iberoamericana para la investigación y el desarrollo educativo, 9(18), 868896. https://doi.org/10.23913/ride.v9i18.480

Mo, Y., y Singh, K. (2015). Parents' Relationships and Involvement: Effects on Students' School Engagement and Performance. RMLE Online, 31(10), 111. https://doi.org/10.1080/19404476.2008.11462053

Morgado, J., Sousa, J., y Pacheco, J. (2020). Transformações educativas em tempos de pandemia: do confinamento social ao isolamento curricular. Praxis Educactiva, 15. https://doi.org/10.5212/PraxEduc.v.15.16197.062

Naranjo, M., Morales, I., y Ron, R. (2020). Efectos de la pandemia en la familia y en la sociedad ecuatoriana. Res Non Verba Revista Científica, 10(2), 115-135. http://revistas.ecotec.edu.ec/index.php/rnv/article/view/417

Park, N. (2004). El papel del bienestar subjetivo en el desarrollo positivo de la juventud. Los anales de la academia estadounidense de ciencias políticas y sociales, 591(1), 25-39. doi:https://doi.org/10.1177/0002716203260078 Paternina, D., y Pereira, M. (2017). Funcionalidad familiar en escolares con conductas de riesgo psicosocial en una institución educativa de sincelejo. Salud Uninorte, 33 (3) 429- 437, 33(3), 429-437. https://search.proquest.com/scholarly-journals/funcionalidad-familiar-enescolares-con/docview/1984766447/se-2?accountid=37408 Pereira, G., y Rivas, J. (2019). Padres y madres: actores indispensables para el éxito académico de los niños. Educación Unisinos, 23(3), 471-487. doi:10.4013/edu.2019.233.06 
Razeto, A. (2018). Estrategias para promover la participación de familias en la educación de niños en escuelas chilenas. Educação e Pesquisa, 44. https://dx.doi.org/10.4067/S0718-07052016000200026

Renta, A., Aubert, A., y Tierno-García, J. (2019). Influencia de la formación de familiares en la motivación del alumnado en riesgo de exclusión social. Revista Mexicana De Investigación Educativa, 24(81), 481-505. https://search.proquest.com/scholarly-journals/influencia-de-la-formaciónfamiliares-en/docview/2263217504/se-2?accountid=37408

Ribeiro, D. y Andrade, A. (2006). A assimetria na relação entre família e escola pública. Paidéia (Ribeirão Preto), 16(35), 385-394. https://doi.org/10.1590/S0103-863X2006000300009

Rodríguez, D., y Vasconcelos, S. (2019). Práticas de integração famíliaescola como preditoras do desempenho escolar de alunos. Psicologia: Ciência e Profissão, 39(e186361), 1-13. https://doi.org/10.1590/19823703003186361

Romagnoli, C., y Gallargo, G. (2018). Alianza Efectiva Familia Escuela: Para promover el desarrollo intelectual, emocional, social y ético de los estudiantes. Centro de recursos digitales. Educarchile: https://centroderecursos.educarchile.cl/handle/20.500.12246/53167

Romero, M. (2020). ¿Qué pasa en las islas? Jóvenes y experiencias formativas en contexto de pandemia y bajante del río Paraná. Millcayac - Revista Digital de Ciencias $\quad$ Sociales, $\quad$ 7(13), 367-388. http://revistas.uncuyo.edu.ar/ojs/index.php/millca-digital/article/view/3536 Ruíz, G. (2020). COVID-19: Pensar la educación en un escenario inédito. Revista Mexicana De Investigación Educativa, 25(85), 229-237. https://search.proquest.com/scholarly-journals/covid-19-pensar-laeducación-en-un-escenario/docview/2424115820/se-2?accountid=37408 
Russo, K., Magnan, M.-O., y Soares, R. (2020). A pandemia que amplia as desigualdades: a Covid-19 e o sistema educativo de Quebec/Canadá. Práxis Educativa (Brasil), 1 1-28. https://doi.org/10.5212/PraxEduc.v.15.15915.073

Ruvalcaba-Romero, N., Orozco-Solis, M., Gallegos-Guajardo, J., y NavaFuerte, J. (2018). Relaciones escolares, comunicación con padres y prosocialidad como predictores de emociones positivas. Liberabit, 24(2), 183-193. https://doi.org/10.24265/liberabit.2018.v24n2.02

Sarmiento, P., y Zapata, M. (2014). Modelo conceptual sobre la participación de la familia en la escuela: un estudio cualitativo en cuatro localidades del Perú. GRADE Group for the Analysis of Development. https://nbnresolving.org/urn:nbn:de:0168-ssoar-56546-2

Scalabrin, A., y Côco, V. (2020). Educación de la primera infancia, políticas gubernamentales y movilizaciones en tiempos de pandemia. Praxis Educativa, 15(1), 1-15. https://doi.org/10.5212/PraxEduc.v.15.16266.088 Tarabini, A. (2020). ¿Para qué sirve la escuela? Reflexiones sociológicas en tiempos de pandemia global. Revista de Sociología de la Educación - RASE, 13(2), 145-155. http://dx.doi.org/10.7203/RASE.13.2.17135

Tarazona, A., Díaz, T., García, M., y Hernández, Á. (2016). La integración escuela familia desde una visión ecuatoriana. Enfermeria Investiga. Revista científica indexada y arbitrada, 1(4), 176-180. https://revistas.uta.edu.ec/erevista/index.php/enfi/article/view/806

Topor, D., Keane, S., Shelton, T., y Calkins, S. (2010). Parent Involvement and Student Academic Performance: A Multiple Mediational Analysis. Journal of Prevention \& Intervention in the Community, 38(3), 183-197. https://doi.org/10.1080/10852352.2010.486297

Valero-Cedeño, N., Castillo-Matute, A., Rodríguez-Pincay, R., PadillaHidalgo, M., y Cabrera-Hernández, M. (2020). Retos de la educación virtual 
en el proceso enseñanza aprendizaje durante la pandemia de Covid-19. Dominio de las ciencias, 6(4), 1201-1220. https://dominiodelasciencias.com/ojs/index.php/es/article/view/1530 Vázquez, M., Bonilla, W., y Acosta, L. (2020). La educación fuera de la escuela en época de pandemia por Covid 19. Experiencias de alumnos y padres de familia. Revista Electrónica Sobre Cuerpos Académicos y Grupos de Investigación, $7(14)$ 111-134. https://cagi.org.mx/index.php/CAGI/article/view/213 Velásquez, L. (2020). ¿Virtualizar o precarizar? consecuencias de la pandemia. $\quad E l \quad$ Cotidiano, 35(221), 61-67. https://search.proquest.com/scholarly-journals/virtualizar-o-precarizarconsecuencias-de-la/docview/2462681051/se-2 accountid=37408 Villalobos, J., Flórez, G., y Londoño, D. (2017). La escuela y la familia en relación con el alcance del logro académico. La experiencia de la Institución Educativa Antonio José de Sucre de Itagüí (Antioquia). Aletheia. Revista de desarrollo humano, educativo y social contemporáneo, 9(1), 58-75. http://www.scielo.org.co/scielo.php?pid=s214503662017000100058\&script=sci_abstract\&tlng=pt Vygotsky, L. (1995). Pensamiento y lenguaje. Teoría del desarrollo cultural de las funciones psíquicas. Fausto.

Wilder, S. (2014). Effects of parental involvement on academic achievement: a meta-synthesis. Educational Review, 66(3), 377-397. https://doi.org/10.1080/00131911.2013.780009 
Tabla 1

Dimensión: Los padres de familia y el aprendizaje de sus hijos

\begin{tabular}{|c|c|c|c|}
\hline Año & Autor(es) /País & Título & Objetivo \\
\hline 2020 & $\begin{array}{l}\text { Cabrera, L. } \\
\text { (España) }\end{array}$ & $\begin{array}{l}\text { Efectos del coronavirus en el } \\
\text { sistema de enseñanza: aumenta } \\
\text { la desigualdad de } \\
\text { oportunidades educativas en } \\
\text { España }\end{array}$ & $\begin{array}{l}\text { Analizar la realidad de los jóvenes en } \\
\text { sus contextos familiares de } \\
\text { confinamiento y sus repercusiones } \\
\text { en el aprendizaje. }\end{array}$ \\
\hline 2020 & $\begin{array}{l}\text { Kohan, W. } \\
\text { (Brasil) }\end{array}$ & $\begin{array}{l}\text { Tempos da escola em tempo de } \\
\text { pandemia e necropolítica. }\end{array}$ & $\begin{array}{l}\text { Describir la forma como el gobierno } \\
\text { desarrolla una necropolítica, durante } \\
\text { pandemia del COVID-19 y cómo } \\
\text { afectó a la escuela y educación de } \\
\text { Brasil. }\end{array}$ \\
\hline 2020 & $\begin{array}{l}\text { Valero-Cedeño, N., } \\
\text { Castillo-Matute, A., } \\
\text { Rodríguez-Pincay, R., } \\
\text { Padilla-Hidalgo, M., y } \\
\text { Cabrera-Hernández, } \\
\text { M. (Ecuador) }\end{array}$ & $\begin{array}{l}\text { Retos de la educación virtual en } \\
\text { el proceso enseñanza } \\
\text { aprendizaje durante la } \\
\text { pandemia de Covid-19. }\end{array}$ & $\begin{array}{l}\text { Analizar los retos que la educación } \\
\text { virtual ha representado en el } \\
\text { proceso de enseñanza aprendizaje } \\
\text { durante la pandemia. }\end{array}$ \\
\hline 2020 & $\begin{array}{l}\text { Vázquez, M., Bonilla, } \\
\text { W., y Acosta, L. } \\
\text { (México) }\end{array}$ & $\begin{array}{l}\text { La educación fuera de la } \\
\text { escuela en época de pandemia } \\
\text { por Covid 19. Experiencias de } \\
\text { alumnos y padres de familia. }\end{array}$ & $\begin{array}{l}\text { Conocer las vivencias, los } \\
\text { sentimientos, así como los estilos de } \\
\text { trabajo de los padres de familia y de } \\
\text { los alumnos en torno a la educación } \\
\text { escolarizada durante el } \\
\text { confinamiento. }\end{array}$ \\
\hline 2019 & $\begin{array}{l}\text { Mayorquín, E., y } \\
\text { Zaldívar, A. } \\
\text { (México) }\end{array}$ & $\begin{array}{l}\text { Participación de los padres en el } \\
\text { rendimiento académico de } \\
\text { alumnos de primaria. }\end{array}$ & $\begin{array}{l}\text { Analizar las características del apoyo } \\
\text { presentado por los padres de familia } \\
\text { y su relación con el aprovechamiento } \\
\text { escolar de los estudiantes. }\end{array}$ \\
\hline 2019 & $\begin{array}{l}\text { Renta, A., Aubert, A., } \\
\text { y Tierno-García, J. } \\
\text { (México) }\end{array}$ & $\begin{array}{l}\text { Influencia de la formación de } \\
\text { familiares en la motivación del } \\
\text { alumnado en riesgo de } \\
\text { exclusión social. }\end{array}$ & $\begin{array}{l}\text { Exponer evidencias científicas sobre } \\
\text { el aumento de la motivación de los } \\
\text { estudiantes en riesgo de exclusión a } \\
\text { través de la participación de sus } \\
\text { familiares en programas de } \\
\text { formación en centros escolares. }\end{array}$ \\
\hline 2017 & $\begin{array}{l}\text { Villalobos, J., Flórez, } \\
\text { G., y Londoño, D. } \\
\text { (Colombia) }\end{array}$ & $\begin{array}{l}\text { La escuela y la familia en } \\
\text { relación con el alcance del logro } \\
\text { académico. La experiencia de la } \\
\text { Institución Educativa Antonio } \\
\text { José de Sucre de Itagüí }\end{array}$ & $\begin{array}{l}\text { Comprender la incidencia del } \\
\text { acompañamiento familiar en } \\
\text { relación con el logro académico en } \\
\text { los estudiantes. }\end{array}$ \\
\hline 2016 & $\begin{array}{l}\text { Tarazona, A., Díaz, T., } \\
\text { García, M., y } \\
\text { Hernández, Á. } \\
\text { (Ecuador) }\end{array}$ & $\begin{array}{l}\text { La integración escuela familia } \\
\text { desde una visión ecuatoriana. }\end{array}$ & $\begin{array}{l}\text { Analizar la interrelación familia } \\
\text { escuela en el Ecuador, a través de } \\
\text { una comparativa con los marcos de }\end{array}$ \\
\hline
\end{tabular}




\section{Tabla 2}

\section{Dimensión: La comunicación entre la escuela y la familia}

\begin{tabular}{|c|c|c|c|}
\hline Año & Autor(es) & Título & Objetivo \\
\hline 2020 & $\begin{array}{l}\text { Arnaiz, P., Y } \\
\text { Chamseddine, M. } \\
\text { (España) }\end{array}$ & $\begin{array}{l}\text { Relación entre familias } \\
\text { migrantes y profesorado: un } \\
\text { camino en construcción }\end{array}$ & $\begin{array}{l}\text { Describir el conocimiento del centro que } \\
\text { poseen los progenitores, el conocimiento } \\
\text { del profesorado sobre la vida del } \\
\text { alumnado y el tipo de relaciones } \\
\text { personales que mantienen ambas partes. }\end{array}$ \\
\hline 2020 & $\begin{array}{l}\text { Garduño, T. } \\
\text { (México) }\end{array}$ & $\begin{array}{l}\text { La pandemia y la utopía: hacia } \\
\text { nuevos paradigmas } \\
\text { educativos. }\end{array}$ & $\begin{array}{l}\text { Analizar la respuesta de las autoridades } \\
\text { educativas ante la pandemia. }\end{array}$ \\
\hline 2020 & $\begin{array}{l}\text { Hurtado, F. } \\
\text { (Venezuela) }\end{array}$ & $\begin{array}{l}\text { La educación en tiempos de } \\
\text { pandemia: los desafíos de la } \\
\text { escuela del siglo XXI. }\end{array}$ & $\begin{array}{l}\text { Identificar los desafíos de la escuela } \\
\text { durante la pandemia COVID-19, } \\
\text { principalmente en las tecnologías de la } \\
\text { información y comunicación. }\end{array}$ \\
\hline 2020 & $\begin{array}{l}\text { Jarquín, M. } \\
\text { (Mexico) }\end{array}$ & $\begin{array}{l}\text { Educación en tiempos virales: } \\
\text { SARS CoV-2, sistemas } \\
\text { educativos y nueva escuela } \\
\text { mexicana. }\end{array}$ & $\begin{array}{l}\text { Mostrar las principales implicaciones y } \\
\text { repercusiones que la pandemia ha } \\
\text { generado en los sistemas educativos a } \\
\text { nivel global. }\end{array}$ \\
\hline 2020 & $\begin{array}{l}\text { Morgado, J., } \\
\text { Sousa, J., y } \\
\text { Pacheco, J. } \\
\text { (Brasil) }\end{array}$ & $\begin{array}{l}\text { Transformações educativas } \\
\text { em tempos de pandemia: do } \\
\text { confinamento social ao } \\
\text { isolamento curricular. }\end{array}$ & $\begin{array}{l}\text { Analizar el cambio de paradigma y el } \\
\text { papel de las tecnologías digitales en las } \\
\text { actividades de enseñanza a distancia y sus } \\
\text { reflejos en términos curriculares. }\end{array}$ \\
\hline 2020 & $\begin{array}{l}\text { Naranjo, M., } \\
\text { Morales, I., y Ron, } \\
\text { R. (Ecuador) } \\
\end{array}$ & $\begin{array}{l}\text { Efectos de la pandemia en la } \\
\text { familia y en la sociedad } \\
\text { ecuatoriana. }\end{array}$ & $\begin{array}{l}\text { Demostrar los efectos de la pandemia en } \\
\text { la sociedad ecuatoriana }\end{array}$ \\
\hline 2020 & $\begin{array}{l}\text { Ruíz, G. } \\
\text { (Mexico) }\end{array}$ & $\begin{array}{l}\text { COVID-19: Pensar la } \\
\text { educación en un escenario } \\
\text { inédito. }\end{array}$ & $\begin{array}{l}\text { Reflexionar sobre la relación entre la } \\
\text { investigación educativa, la práctica } \\
\text { profesional y la toma de decisiones } \\
\text { durante la pandemia. }\end{array}$ \\
\hline 2020 & $\begin{array}{l}\text { Russo, K., } \\
\text { Magnan, M.-O., y } \\
\text { Soares, R. } \\
\text { (Canadá) }\end{array}$ & $\begin{array}{l}\text { A pandemia que amplia as } \\
\text { desigualdades: a Covid-19 e o } \\
\text { sistema educativo de } \\
\text { Quebec/Canadá. }\end{array}$ & $\begin{array}{l}\text { Identificar los diferentes impactos y } \\
\text { reordenamientos en la educación escolar } \\
\text { durante la crisis sanitaria y discutir cómo } \\
\text { se ampliaron las desigualdades } \\
\text { educativas. }\end{array}$ \\
\hline 2020 & $\begin{array}{l}\text { Scalabrin, A., y } \\
\text { Côco, V. } \\
\text { (Brasil) }\end{array}$ & $\begin{array}{l}\text { Educación de la primera } \\
\text { infancia, políticas } \\
\text { gubernamentales y }\end{array}$ & $\begin{array}{l}\text { Abordar las políticas gubernamentales } \\
\text { relacionadas con la Educación Infantil, } \\
\text { prestando atención a la realidad de los } \\
\text { niños, sus familias y profesionales. }\end{array}$ \\
\hline
\end{tabular}




\begin{tabular}{|c|c|c|c|}
\hline & & $\begin{array}{l}\text { movilizaciones en tiempos de } \\
\text { pandemia. }\end{array}$ & \\
\hline 2020 & $\begin{array}{l}\text { Tarabini, A. } \\
\text { (España) }\end{array}$ & $\begin{array}{l}\text { ¿Para qué sirve la escuela? } \\
\text { Reflexiones sociológicas en } \\
\text { tiempos de pandemia global. }\end{array}$ & $\begin{array}{l}\text { Reflexionar sobre el papel de la escuela } \\
\text { desde un enfoque de la justicia educativa } \\
\text { y social. }\end{array}$ \\
\hline 2020 & $\begin{array}{l}\text { Velásquez, L. } \\
\text { (Mexico) }\end{array}$ & $\begin{array}{l}\text { ¿Virtualizar o precarizar? } \\
\text { Consecuencias de la } \\
\text { pandemia. }\end{array}$ & $\begin{array}{l}\text { Analizar la brecha de exclusión social y } \\
\text { digital en la escuela privada y pública } \\
\text { mexicana en los tiempos de pandemia. }\end{array}$ \\
\hline 2018 & $\begin{array}{l}\text { Lupion, G. } \\
\text { (Brasil) }\end{array}$ & $\begin{array}{l}\text { A relação escola-família e a } \\
\text { organização do trabalho } \\
\text { pedagógico na educação } \\
\text { infantil. }\end{array}$ & $\begin{array}{l}\text { Dar conocer las diferentes formas para el } \\
\text { trabajo con las familias y reforzar la } \\
\text { necesidad de la escuela en mantener sus } \\
\text { conocimientos actualizados sobre los } \\
\text { niños y sus familias. }\end{array}$ \\
\hline 2018 & $\begin{array}{l}\text { Macia, M. } \\
\text { (España) }\end{array}$ & $\begin{array}{l}\text { Aproximación teórica a la } \\
\text { comunicación familia-escuela: } \\
\text { Estrategias de mejora. }\end{array}$ & $\begin{array}{l}\text { Presentar las grandes líneas estratégicas } \\
\text { para optimizar la comunicación con las } \\
\text { familias y dotar a los maestros de } \\
\text { herramientas básicas de acuerdo a sus } \\
\text { características y necesidades }\end{array}$ \\
\hline 2016 & $\begin{array}{l}\text { Lv, B., Zhou, H., } \\
\text { Guo, X., Liu, C., } \\
\text { Liu, Z., y Luo, L. } \\
\text { (China) }\end{array}$ & $\begin{array}{l}\text { The relationship between } \\
\text { academic achievement and } \\
\text { the emotional well-being of } \\
\text { elementary school children in } \\
\text { China: The moderating role of } \\
\text { parent-school } \\
\text { communication. }\end{array}$ & $\begin{array}{l}\text { Investigar la relación entre el rendimiento } \\
\text { académico y el bienestar emocional } \\
\text { emocional de los niños de la escuela } \\
\text { primaria y el efecto moderador de la } \\
\text { comunicación entre padres y la escuela. }\end{array}$ \\
\hline
\end{tabular}

Tabla 3

Dimensión: Participación de la familia en la gestión y actividades de la escuela

\begin{tabular}{llll}
\hline Año & \multicolumn{1}{c}{ Autor(es) /País } & \multicolumn{1}{c}{ Título } & \multicolumn{1}{c}{ Objetivo } \\
\hline 2020 & $\begin{array}{l}\text { Arnaiz, P., y } \\
\text { Chamseddine, M. } \\
\text { (España) }\end{array}$ & $\begin{array}{l}\text { Relación entre familias } \\
\text { migrantes y profesorado: un } \\
\text { camino en construcción }\end{array}$ & $\begin{array}{l}\text { Describir el conocimiento del centro que } \\
\text { poseen los progenitores, el } \\
\text { conocimiento del profesorado sobre la } \\
\text { vida del alumnado y el tipo de relaciones } \\
\text { personales que mantienen ambas } \\
\text { partes. }\end{array}$ \\
\hline 2020 & $\begin{array}{l}\text { Belmonte, M., y } \\
\text { Bernárdez-Gómez, } \\
\text { A. (España) }\end{array}$ & $\begin{array}{l}\text { Respuesta social al estado del } \\
\text { aislamiento por coronavirus, } \\
\text { percepciones sobre la } \\
\text { educación }\end{array}$ & $\begin{array}{l}\text { Recopilar y contrastar las percepciones } \\
\text { sobre la educación, formal o no formal, } \\
\text { llevada a cabo en los hogares, a raíz del } \\
\text { confinamiento. }\end{array}$ \\
\hline 2020 & $\begin{array}{l}\text { Hurtado, F. } \\
\text { (Venezuela) }\end{array}$ & $\begin{array}{l}\text { La educación en tiempos de } \\
\text { pandemia: los desafíos de la } \\
\text { escuela del siglo XXI. }\end{array}$ & $\begin{array}{l}\text { Identificar los desafíos de la escuela } \\
\text { durante la pandemia COVID-19, }\end{array}$ \\
\hline
\end{tabular}




\begin{tabular}{|c|c|c|c|}
\hline & & & $\begin{array}{l}\text { principalmente en las tecnologías de la } \\
\text { información y comunicación }\end{array}$ \\
\hline 2020 & $\begin{array}{l}\text { Romero, M. } \\
\text { (Argentina) }\end{array}$ & $\begin{array}{l}\text { ¿Qué pasa en las islas? Jóvenes } \\
\text { y experiencias formativas en } \\
\text { contexto de pandemia y } \\
\text { bajante del río Paraná. }\end{array}$ & $\begin{array}{l}\text { Contextualizar las vivencias actuales que } \\
\text { permean y constituyen las experiencias } \\
\text { formativas de familias y estudiantes. }\end{array}$ \\
\hline 2019 & $\begin{array}{l}\text { Pereira, E. } \\
\text { Rivas, J } \\
\text { (Brasil) }\end{array}$ & $\begin{array}{l}\text { Padres y madres: actores } \\
\text { indispensables al éxito } \\
\text { académico de los niños. }\end{array}$ & $\begin{array}{l}\text { Conocer las opiniones de los niños y } \\
\text { padres de familia sobre su participación } \\
\text { de estos en su vida escolar. }\end{array}$ \\
\hline 2018 & $\begin{array}{l}\text { Razeto, A. } \\
\text { (Chile) }\end{array}$ & $\begin{array}{l}\text { Estrategias para promover la } \\
\text { participación de familias en la } \\
\text { educación de niños en escuelas } \\
\text { chilenas. }\end{array}$ & $\begin{array}{l}\text { Reflexionar acerca del potencial que } \\
\text { presenta la estrategia de promoción de } \\
\text { la participación de los padres en la } \\
\text { educación de los hijos para favorecer su } \\
\text { éxito académico. }\end{array}$ \\
\hline 2018 & $\begin{array}{l}\text { Ruvalcaba- } \\
\text { Romero, N., } \\
\text { Orozco-Solis, M., } \\
\text { Gallegos-Guajardo, } \\
\text { J., y Nava-Fuerte, J. } \\
\text { (México) }\end{array}$ & $\begin{array}{l}\text { Relaciones escolares, } \\
\text { comunicación con padres y } \\
\text { prosocialidad como } \\
\text { predictores de emociones } \\
\text { positivas. }\end{array}$ & $\begin{array}{l}\text { Identificar el nivel predictivo de las } \\
\text { relaciones entre compañeros de clase, la } \\
\text { comunicación afectiva con los padres y } \\
\text { la generación de conductas prosociales } \\
\text { sobre la experimentación de emociones } \\
\text { positivas en adolescentes. }\end{array}$ \\
\hline 2017 & $\begin{array}{l}\text { León, V. y } \\
\text { Fernández, M. } \\
\text { (España) }\end{array}$ & $\begin{array}{l}\text { Diseño y validación de un } \\
\text { instrumento para evaluar la } \\
\text { participación de las familias en } \\
\text { los centros educativos }\end{array}$ & $\begin{array}{l}\text { Diseñar y validar un instrumento para } \\
\text { evaluar la participación de las familias } \\
\text { en los centros educativos }\end{array}$ \\
\hline 2015 & $\begin{array}{l}\text { Mo, Y., y Singh, K. } \\
\text { (USA) }\end{array}$ & $\begin{array}{l}\text { Parents' Relationships and } \\
\text { Involvement: Effects on } \\
\text { Students' School Engagement } \\
\text { and Performance. }\end{array}$ & $\begin{array}{l}\text { Examinar el efecto de las relaciones y la } \\
\text { participación de los padres en el } \\
\text { compromiso cognitivo, emocional y } \\
\text { conductual de los estudiantes en la } \\
\text { escuela y el rendimiento escolar. }\end{array}$ \\
\hline 2010 & $\begin{array}{l}\text { Domingo, J., } \\
\text { Martos, M., y } \\
\text { Domingo, L. } \\
\text { (España) }\end{array}$ & $\begin{array}{l}\text { Colaboración familia - escuela } \\
\text { en España: retos y realidades. }\end{array}$ & $\begin{array}{l}\text { Proponer líneas de trabajo para mejorar } \\
\text { la relación escuela - familia }\end{array}$ \\
\hline
\end{tabular}

\section{Figura 1}

Participacipacion de los padres de familia en el aprendizaje de los estudiantes 


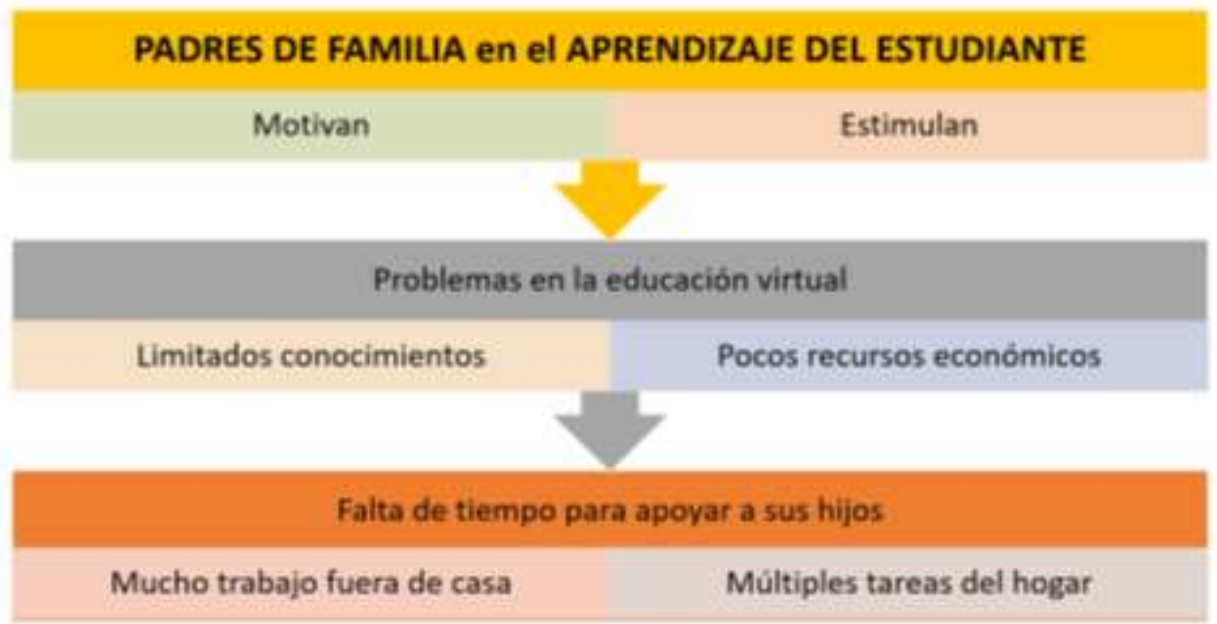

\section{Figura 2}

La comunicación entre la escuela y los padres de familia

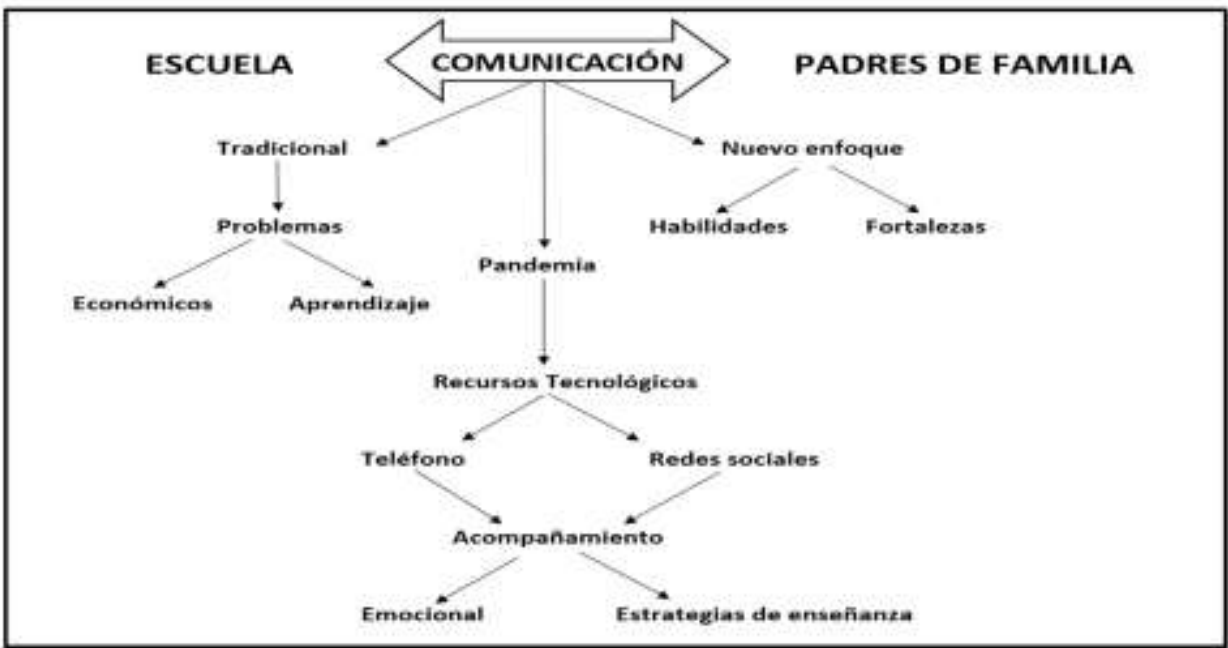

Figura 3

Participación de los padres de familia en las actividades de la escuela 


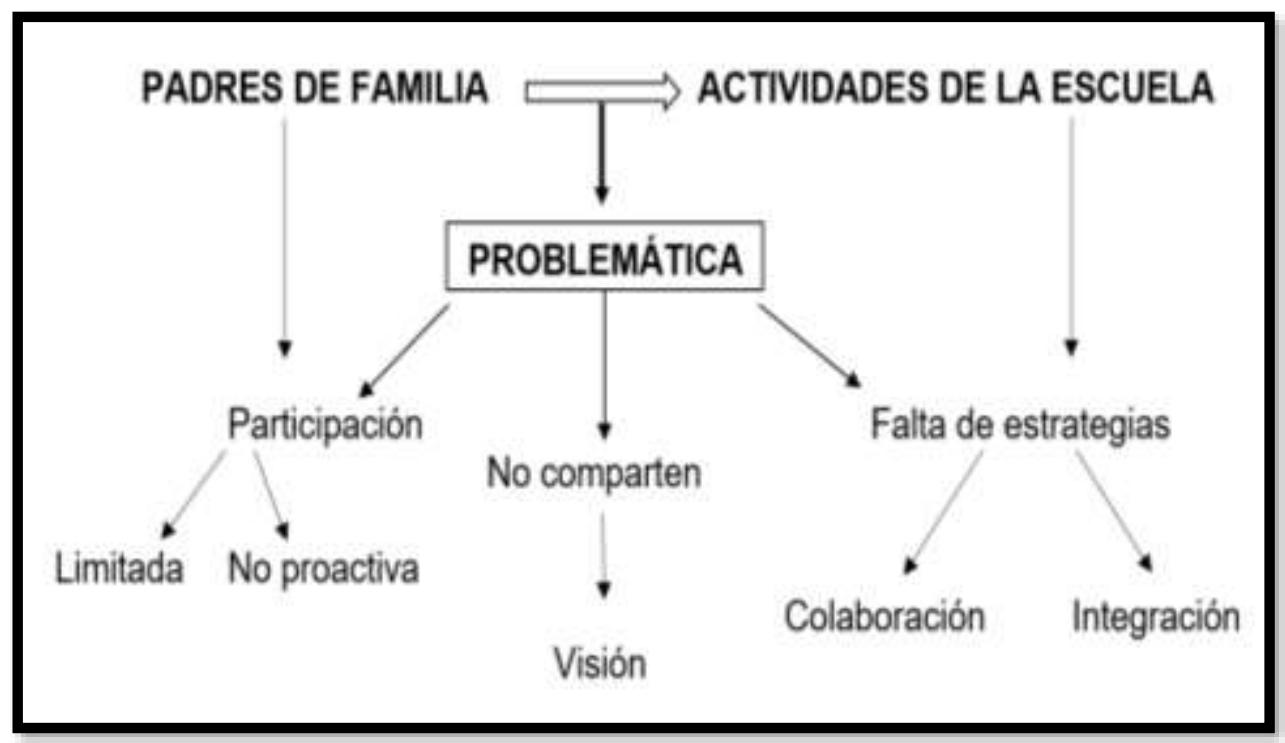

